\title{
Screening of Health-Associated Oral Bacteria for Anticancer Properties in vitro
}

\author{
Divyashri Baraniya ${ }^{1}$, Vinay Jain ${ }^{1}$, Ronald Lucarelli ${ }^{2}$, Vincent Tam $^{2}$, Lisa Vanderveer ${ }^{3}$, \\ Sumant Puri ${ }^{1}$, Maobin Yang ${ }^{4}$ and Nezar Noor Al-hebshi ${ }^{1,3 *}$ \\ 'Oral Microbiome Research Laboratory, Department of Oral Health Sciences, Maurice H. Kornberg School of Dentistry, \\ Temple University, Philadelphia, PA, United States, ${ }^{2}$ Department of Microbiology and Immunology, Lewis Katz School of \\ Medicine, Temple University, Philadelphia, PA, United States, ${ }^{3}$ Fox Chase Cancer Center, Temple University Health System, \\ Philadelphia, PA, United States, ${ }^{4}$ Regenerative Research Laboratory, Department of Endodontology, Maurice H. Kornberg \\ School of Dentistry, Temple University, Philadelphia, PA, United States
}

\section{OPEN ACCESS}

Edited by:

Yinduo Ji,

University of Minnesota Twin Cities,

United States

Reviewed by:

Richard Lamont,

University of Louisville, United States

Oleh Andrukhov,

University Dental Clinic Vienna, Austria

*Correspondence:

Nezar Noor Al-hebshi alhebshi@temple.edu

Specialty section:

This article was submitted to

Bacteria and Host,

a section of the journa

Frontiers in Cellular and Infection

Microbiology

Received: 24 June 2020 Accepted: 02 September 2020

Published: 06 October 2020

Citation:

Baraniya D, Jain V, Lucarelli R, Tam V, Vanderveer L, Puri S, Yang $M$ and Al-hebshi NN (2020) Screening of Health-Associated Oral Bacteria for Anticancer Properties in vitro. Front. Cell. Infect. Microbiol. 10:575656.

doi: 10.3389/fcimb.2020.575656
While extensive literature exists about the role of oral bacterial pathogens like Porphyromonas gingivalis and Fusobacterium nucleatum in oral squamous cell carcinoma (OSCC), the role of health-associated species has been largely unexplored. In this study, we assessed the effect of Streptococcus mitis, Rothia mucilaginosa, Neisseria flavescens, Haemophilus parainfluenzae, Lautropia mirabilis, and Veillonella parvula on proliferation and expression of marker genes (IL-6, TNF- $\alpha$, MMP3, CD36, CCD1, and NANOG) in OSCC cell lines CAL27, SCC25, and SCC4. Porphyromonas gingivalis was included as a pathogenic control. Both bacterial lysates (3 concentrations) and live cells (3 MOls) were tested. S. mitis, $H$. parainfluenzae, and $N$. flavescens resulted in substantial, dose-dependent reduction of proliferation, which was found to be mediated by $\mathrm{H}_{2} \mathrm{O}_{2}$ for the former and intracellular infection in the latter two species. However, only $H$. parainfluenzae showed differential antiproliferative effect against the cancer cell lines vs. the normal control (TIGKs). In the gene expression assays, the health-associated species mostly downregulated CD36, a gene that plays an important role in tumor growth and metastasis, while $P$. gingivalis upregulated it. IL6 and TNF expression, on the other hand, was upregulated by almost all species, particularly the Gram-negatives including P. gingivalis. The effect on other genes was less evident and varied significantly by cell line. This exploratory study is the first insight into how health-associated bacteria may interact with OSCC. Further studies to explore whether the observed effects may have implications for the prevention or treatment of oral cancer are warranted.

Keywords: bacteria, carcinoma, cell line, cell proliferation, mouth, coculture techniques, gene expression

\section{INTRODUCTION}

Oral cancer, predominantly oral squamous cell carcinoma (OSCC), continues to be a major global health burden, with poor prognosis and a 5-year survival rate of $<50 \%$. It accounts for 354,864 new cases and 177,384 deaths globally each year (Bray et al., 2018). There is a male predilection, and the tongue is the most commonly affected subsite (Siegel et al., 2017). Use of smoked and smokeless forms of tobacco, alcohol consumption, and betelnut chewing are the major risk factors (Gupta et al., 2016), whereas 2-6\% of the cases are attributed to human papilloma virus (HPV) (Lingen et al., 2013). However, about $15 \%$ of all OSCC cases are not associated with any of the known risk factors (Chocolatewala et al., 2010). 
Recently, there has been increasing interest in the potential role of the microbiome in oral cancer. In a plethora of clinical studies, analyses of samples from OSCC patients have revealed significant differences in microbiome composition and function between tumor and control samples, with certain taxa quite consistently showing association with OSCC across studies (Perera et al., 2016; Al-Hebshi et al., 2019). Of these, Fusobacterium nucleatum and Porphyromonas gingivalis have received the most attention, with large number of studies that characterized their carcinogenic properties in vitro and in in vivo models (Fitzsimonds et al., 2020). However, in focusing on exploring the role of species that are enriched in the tumors, i.e., the pathobionts, researchers seem to have largely ignored the health-associated species that in fact may be playing a protective role against oral cancer. Indeed, Ohshima et al. (2019) have very recently found Streptococcus gordonii, a health-associated species, to antagonize the pro-cancer phenotypes induced by $P$. gingivalis, highlighting the need to explore the other side of the relationship between the microbiome and oral cancer.

A number of bacterial taxa have consistently been found to be more abundant in samples from healthy subjects compared to those from cancer/precancer patients. These include Streptococcus spp. such as Streptococcus mitis (Pushalkar et al., 2012; Schmidt et al., 2014; Al-Hebshi et al., 2017; Amer et al., 2017), Haemophilus spp., mainly Hemophilus parainfluenzae (AlHebshi et al., 2017; Guerrero-Preston et al., 2017; Perera et al., 2017), Neisseria spp. (Al-Hebshi et al., 2017; Amer et al., 2017; Guerrero-Preston et al., 2017; Hayes et al., 2018), Lautropia spp., mostly L. mirabilis (Al-Hebshi et al., 2017; Amer et al., 2017; Perera et al., 2017; Zhao et al., 2017) Veillonela spp. (Al-Hebshi et al., 2017; Zhao et al., 2017) and Rothia spp. (Schmidt et al., 2014; Al-Hebshi et al., 2017; Zhao et al., 2017), although for the latter there are studies that show an association with cancer (Amer et al., 2017; Mukherjee et al., 2017). One study even found the relative abundances of $S$. mitis, $H$. parainfluenzae, and Veillonella parvula to inversely correlate with oral cancer staging (Yang et al., 2018). None of these presumably health-associated taxa has been screened for its effect on epithelium or OSCC cell lines yet.

The objective of this study was to assess the in vitro effects of a panel of oral health-associated bacterial species, identified from previous microbiome studies, on oral squamous cell carcinoma (OSCC) cell lines. Namely S. mitis, Rothia mucilaginosa, Veillonella parvula, Neisseria flavescens, H. parainfluenzae, and L. mirabilis, were individually screened for their effect on proliferation and gene expression in OSCC cell lines CAL27, SCC25, and SCC4, in comparison with. P. gingivalis as a carcinogenic species. The gene panel included markers of inflammation (IL-6 and TNF- $\alpha$ ), invasion and metastasis (MMP3 and CD36), proliferation (CCND1), and stemness (NANOG).

\section{MATERIALS AND METHODS}

\section{Bacterial Strains and Culture Conditions}

Porphyromonas gingivalis ATCC 33277, Veillonella parvula ATCC 17745, Streptococcus mitis ATCC 49456, Neisseria flavescens ATCC 13120, Hemophilus parainfluenzae NCTC 10665, Rothia mucilaginosa ATCC 49041, and Lautropia mirabilis
ATCC 51599 were obtained from the American Tissue Culture Collection (ATCC) or Public Health England. V. parvula was cultivated in Brain Heart Infusion (BHI) supplemented with 1.5 of $60 \%$ sodium lactate. All other bacteria were cultivated in BHI supplemented with $0.5 \%$ hemin, $0.1 \%$ Vitamin $\mathrm{K}$ and $1 \%$ Isovitalex. $P$. gingivalis and $V$. parvula were grown in anaerobic conditions ( $10 \%$ hydrogen, $10 \% \mathrm{CO}_{2}$, and $80 \%$ nitrogen), $L$. mirabilis was grown aerobically, and the other five bacteria were grown in $5 \% \mathrm{CO}_{2}$. All strains were grown at $37^{\circ} \mathrm{C}$.

\section{Cell Lines and Growth Conditions}

OSCC cell lines SCC4 (RRID: CVCL_1684), SCC25 (RRID: CVCL_1682), and CAL27 (RRID: CVCL_1107), in addition to telomerase immortalized human gingival keratinocytes (TIGKs; RRID: CVCL_M095) as non-cancer control, were obtained from ATCC, with certificates confirming the cells were mycoplasmafree and authenticated by STR. Dulbecco's modified Eagle's medium (DMEM) supplemented with $10 \%$ fetal bovine serum (FBS) and $2.5 \mathrm{mM}$ L-glutamine was used for growing CAL27cell line. A 1:1 mixture of DMEM and Ham's F12 medium containing $2.5 \mathrm{mM}$ L-glutamine, $400 \mathrm{ng} / \mathrm{ml}$ hydrocortisone and 10\% FBS was used for growing cell lines SCC4 and SCC25. Dermal Cell Basal Medium (ATCC PCS-200-030) supplemented with keratinocyte growth kit (ATCC PCS-200-040) was used for growing TIGKs cells. All the cell lines were grown in $5 \% \mathrm{CO}_{2}$ at $37^{\circ} \mathrm{C}$.

\section{Experimental Design}

The study involved testing each of the 7 bacterial strains against each of the 3 cell lines as a lysate at 3 concentrations or in co-culture at 3 multiplicity of infections (MOIs). Parameters assessed were proliferation (counting and ATP assay) and expression of 6 marker genes. All experiments were performed in triplicates for each assay and timepoint. Strains that showed prominent results in the proliferation assay were also tested on non-cancer cell line TIGKs. A total of 1,872 counts, 1,682 ATP assays, 504 RNA extractions, and 3, 982 real-time PCR reactions were performed.

\section{Treatment of OSCC Cells With Bacterial Lysates}

Lysates were prepared from the bacterial strains grown to midlog stage as described in Supplementary Method 1. For the proliferation assay, OSCC cells were suspended in the respective culture media supplemented with penicillin/streptomycin mix at a final concentration of $100 \mathrm{I}$.U./ml and seeded to 48 well plates (TPP, Switzerland) at a density of 7,500 cells/well. For studying gene expression, cells were seeded at 25,000-35,000 cells/well depending on the cell line. The cells were allowed to attach for $24 \mathrm{~h}$ before the lysates were added to a final concentration of 200, 100 , or $50 \mu \mathrm{g} / \mathrm{ml}$ (15 mM HEPES buffer was added to the control wells). Proliferation was assessed at 24,48 , and $72 \mathrm{~h}$ after addition of the lysates, while RNA extraction for gene expression analysis was done at $24 \mathrm{~h}$ of exposure (at which time the cells were $\sim 90 \%$ confluent). 


\section{Co-culture of OSCC Cells With Bacteria}

The bacterial strains were grown to mid log stage as described in Supplementary Method 2. Actively growing bacterial cells were pelleted by centrifugation at 5,000 rpm for $5 \mathrm{~min}$, washed three times in phosphate-buffered saline (PBS), and suspended in DMEM media supplemented with $10 \%$ fetal bovine serum (FBS) and $2.5 \mathrm{mM}$ L-glutamine. They were then added at MOIs of 10,50 , and 100 to the OSCC cells seeded $24 \mathrm{~h}$ before as described above (DMEM media added to control wells). To allow bacterial exposure throughout the experimental period without overgrowing, penicillin/streptomycin mix was included in the culture medium at sub-minimum inhibitory concentration (sub-MIC) which was determined for each bacterium prior to the experiment as described in Supplementary Method 3. Proliferation and gene expression were assessed at the same timepoints as for the lysates experiment.

\section{Cell Proliferation}

This was assessed by cell counting and ATP assay. For counting, cells were harvested from wells using Trypsin-EDTA solution after washing with PBS. After neutralizing trypsin, harvested cells were counted using a Luna-II ${ }^{\mathrm{TM}}$ automated cell counter (Logosbio). ATP was measured using the CellTiter-Glo ${ }^{\circledR} 2.0$ Cell Viability Assay Kit (Promega, USA) according to manufacturer's instructions, and luminescence was measured on a multimode plate reader (Biotek, Synergy HTX).

\section{RNA Extraction and Gene Expression Assays}

The culture medium was removed and stored at $-80^{\circ} \mathrm{C}$ for protein analysis, the cells were washed with PBS, and RNA was isolated using the PureLink RNA extraction kit (Invitrogen, USA) following manufacturer's instructions. SUPERase.In ${ }^{\mathrm{TM}}$ RNase inhibitor (Invitrogen) was added to the extracted RNA at a concentration of $1 \mathrm{U} / \mu \mathrm{l}$ of RNA and contaminating DNA was digested using the Turbo DNA-free ${ }^{\mathrm{TM}}$ kit (Invitrogen). RNA yield and quality were assessed using a Nanodrop (ThermoFisher Scientific, USA). Extracted RNA was directly used in TaqMan ${ }^{\circledR}$ Array Standard Plates (4413266, ThermoFisher Scientific, USA) with predesigned primers/probe sets for human genes TNF- $\alpha$, IL6, CD36, MMP3, CCND1, NANOG, and CASC3, employing a one-step, quantitative, reverse-transcription PCR (one-step qRT-PCR). Specifically, the SuperScript ${ }^{\mathrm{TM}}$ III Platinum ${ }^{\mathrm{TM}}$ OneStep qRT-PCR Kit (ThermoFisher Scientific, USA) was used in $20 \mu \mathrm{l}$ reactions as recommended by the manufacturer, using \%35 ng total RNA as template. Quantification was performed on a Quantstudio TM 3.0 Real-Time PCR system (Applied Biosystems, USA) using the following thermal cycling conditions: cDNA synthesis for $15 \mathrm{~min}$ at $50^{\circ} \mathrm{C}$, initial denaturation at $95^{\circ} \mathrm{C}$ for $2 \mathrm{~min}$ and 40 cycles of denaturation at $90^{\circ} \mathrm{C}$ for $15 \mathrm{~s}$ and extension at $60^{\circ} \mathrm{C}$ for $1 \mathrm{~min}$ with data collection at the end of each extension step. Results were analyzed on QuantStudio 3.0 software using CASC3 gene as internal control. The gene expression levels for each target gene were calculated relative to the negative control (no exposure) using the $2^{\text {(delta delta Ct) }}$ method (Livak and Schmittgen, 2001). The genes' assay manufacturer IDs are shown in Supplementary Method 4.

\section{Statistical Analyses}

For the proliferation assays (counting and ATP), two-way analysis of variance (ANOVA) with time and concentration as variables, followed by Dunnett's multiple comparison (Dunnett, 1955) was used to determine statistical significance of differences between the exposed and control cells. Significance in differences in gene expression and protein levels was sought using multiple $t$ tests comparing each concentration against the control followed by Benjamini et al. (2006) method to control for false discovery rates (FDR). GraphPad Prism 7 was used for statistical analysis and generating the figures.

\section{RESULTS}

\section{Effects on Proliferation-General Findings}

The effect of bacterial lysates on proliferation of the three cell lines as assessed by counting and ATP assay is shown in Supplementary Figures 1-6. With the exception of $S$. mitis, the lysates resulted in no to marginal reduction of proliferation, with SCC4 being the least sensitive. Although the lysates from $H$. parainfluenzae and $N$. flavescens showed a more prominent effect on cell counts (especially in SCC25), that was not confirmed by the ATP assay. The results from the co-culture experiments are presented in Supplementary Figures 7-12. The pathogenic control $P$. gingivalis effect varied from slight decrease to slight increase in proliferation depending on the cell line and nature of exposure (lysate vs. coculture). Only S. mitis, $H$. parainfluenzae and $N$. flavescens resulted in pronounced reduction of proliferation, confirmed by both counting and ATP measurement. These are described in details in the following sections.

\section{Cytotoxicity of S. mitis Is Mediated by $\mathrm{H}_{2} \mathrm{O}_{2}$}

S. mitis lysate led to inhibition of proliferation of CAL27 in dose-dependent manner (Figure 1A); at the highest concentration $(200 \mu \mathrm{g} / \mathrm{ml})$, the counts at $72 \mathrm{~h}$ were lower than baseline, indicating the observed effect was due to cytotoxicity. Proliferation of SCC4 and SCC25 was not affected at similar concentrations, however, treatment with higher concentrations (400 and $600 \mu \mathrm{g} / \mathrm{ml})$ significantly reduced proliferation, but still not to levels seen in CAL27 (Figure 1B). In coculture, $S$. mitis reduced proliferation of all the three OSCC cell lines, but as with the lysates, CAL27 was the most sensitive (Figure 2A). Proliferation of TIGKs was affected similar to CAL27. To test if other Streptococci have similar properties, we cocultured CAL27 with Streptococcus mutans and Streptococcus sanguinis, and found the latter, but not the former, to result in a reduction in proliferation similar to that induced by $S$. mitis (Supplementary Figure 13). S. sanguinis and S. mitis belong the same group of Streptococci which are known produce $\mathrm{H}_{2} \mathrm{O}_{2}$. To find out if the cytotoxic effect of $S$. mitis observed was mediated by $\mathrm{H}_{2} \mathrm{O}_{2}$, we performed a separate set of experiments where 20 or $200 \mathrm{U} / \mathrm{ml}$ of Catalase (Sigma-Aldrich), an enzyme that decomposes $\mathrm{H}_{2} \mathrm{O}_{2}$, was added to CAL27 prior to treatment with $S$. mitis lysate at a concentration of $400 \mu \mathrm{g} / \mathrm{ml}$ or coculture at MOI 100. The addition of catalase inhibited $S$. mitis cytotoxicity (Figures 2B,C) although not entirely, which demonstrated that 

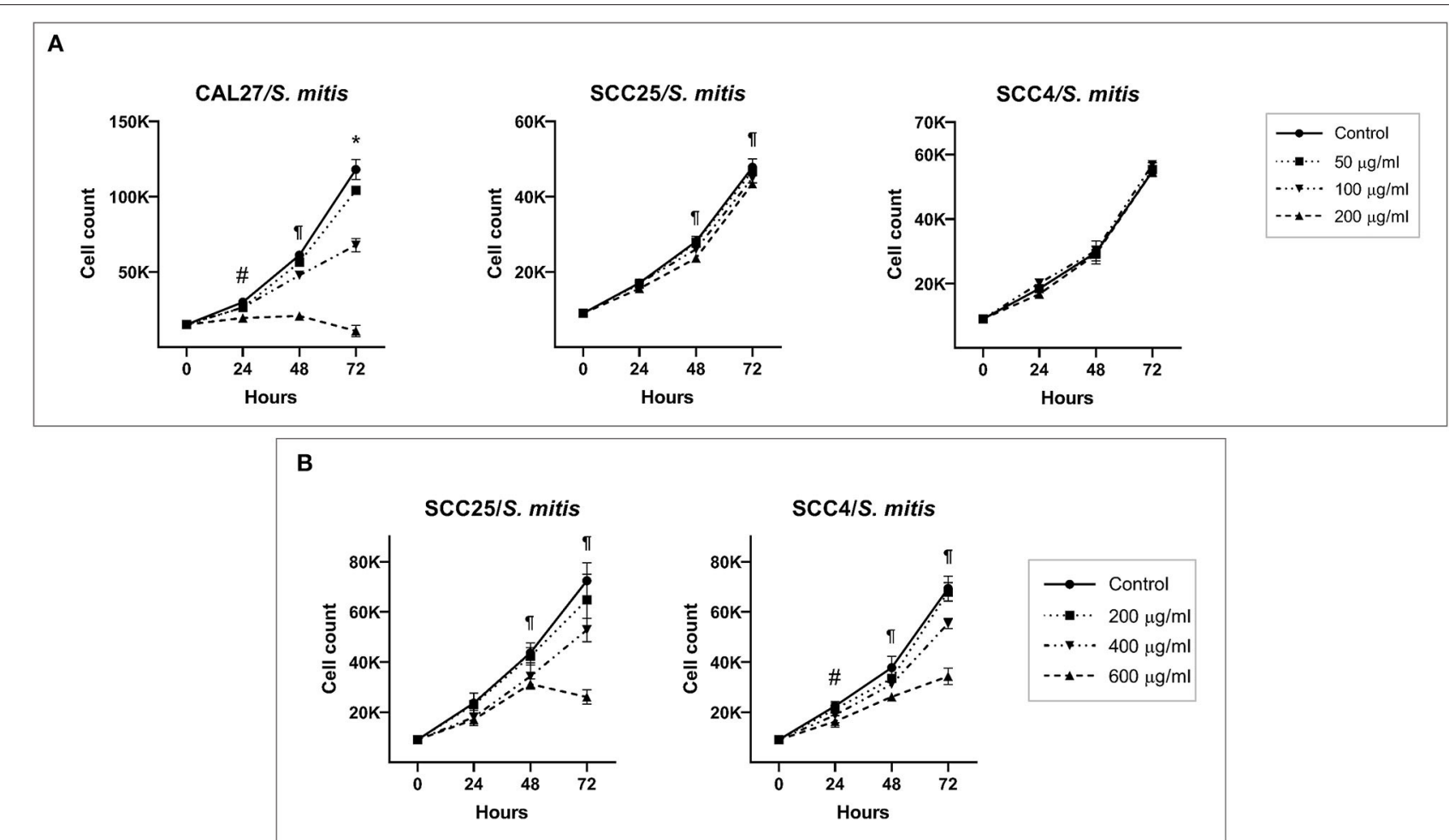

FIGURE 1 | Effect of S. mitis lysate on proliferation of oral cancer cell lines. (A) CAL27, SCC25, and SCC4 were exposed to 50, 100, and 200 $\mu \mathrm{g} / \mathrm{ml}$ of the lysate and counting performed at 24,48 , and $72 \mathrm{~h}$. ${ }^{*}$ Statistically significant $(p \leq 0.05)$ for all concentrations compared to the control; Ilfor $100 \mathrm{and} 200 \mu \mathrm{g} / \mathrm{ml}$; \# for $200 \mu \mathrm{g} / \mathrm{ml}$ only. (B) SCC25 and SCC4 were treated with higher concentrations (400 and $600 \mu \mathrm{g} / \mathrm{ml}$ ) of the lysates. "IStatistically significant $(p \leq 0.05)$ for 400 and $600 \mu \mathrm{g} / \mathrm{ml}$ compared to the control; \# for $600 \mu \mathrm{g} / \mathrm{ml}$ only. The corresponding results from the ATP assay are shown in Supplementary Figures 4-6.

most $S$ mitis cytotoxicity was mediated by $\mathrm{H}_{2} \mathrm{O}_{2}$. To explore the underlying mechanism further, we assessed apoptosis using the Caspase-Glo ${ }^{\circledR}$ 3/7 and RealTime-Glo ${ }^{\mathrm{TM}}$ Annexin V Apoptosis and Necrosis assays (Promega, USA), and found exposure to $S$ mitis at MOI 10 and 50 induces increased caspase activity and loss if cell membrane integrity at 5 and $20 \mathrm{~h}$, respectively (Supplementary Tables 1, 2).

\section{H. parainfluenzae and $N$. flavescens Inhibit Proliferation by Intracellular Infection}

$H$. parainfluenzae and $N$. flavescens caused substantial inhibition of cell proliferation in all the three OSCC cell lines as well as TIGKs in a dose-dependent manner, with CAL27 being the most sensitive (Figure 3). For S. mitis and N. flavescens, reduction in proliferation of TIGKs did not significantly differ from that of the OSCC cell lines (Supplementary Figure 14); however, $H$. parainfluenzae resulted in significantly higher inhibition of OSCC cell lines compared to TIGKs (Figure 4), especially at MOI 50. Interestingly, $N$. flavascens and $H$. parainfluenzae in coculture with the cell lines showed strong growth after $48 \mathrm{~h}$ of inoculation despite presence of subMIC of antibiotics that was otherwise sufficient to control growth in the absence of the cells (results shown for $N$. flavascens with CAL27 and SCC4 in Supplementary Tables 3, 4), which was taken as an evidence of intracellular infection.
To confirm that, we performed intracellular vs. extracellular bacteria staining as described in Supplementary Method 5. Images obtained (Supplementary Figures 15-17) revealed internalization of both bacteria in the three cell lines. This was further confirmed by performing antibiotic protection assays (Supplementary Method 6). The numbers of viable intracellular bacteria recovered from each of the three cell lines are shown in Supplementary Tables 5, 6.

\section{Effect on Gene Expression-General Findings}

Changes in the expression of the six marker genes in response to exposure to the bacterial lysates are shown for the three cell lines in Supplementary Figures 18-20, while the corresponding results from the co-culture experiments are illustrated in Supplementary Figures 21-23. A fold change of $\geq 2$ was used to demarcate pronounced effects. Each cell line showed a unique response. Some changes in gene expression were concentration-dependent while others were concentrationindependent. Overall, the results from the lysate and co-culture experiments were consistent. CCND1 was the least responsive gene in all cell lines, followed by NANOG. Results for MMP3 varied significantly by cell line and the nature of exposure (lysate vs. coculture). More consistent and profound results, which are 

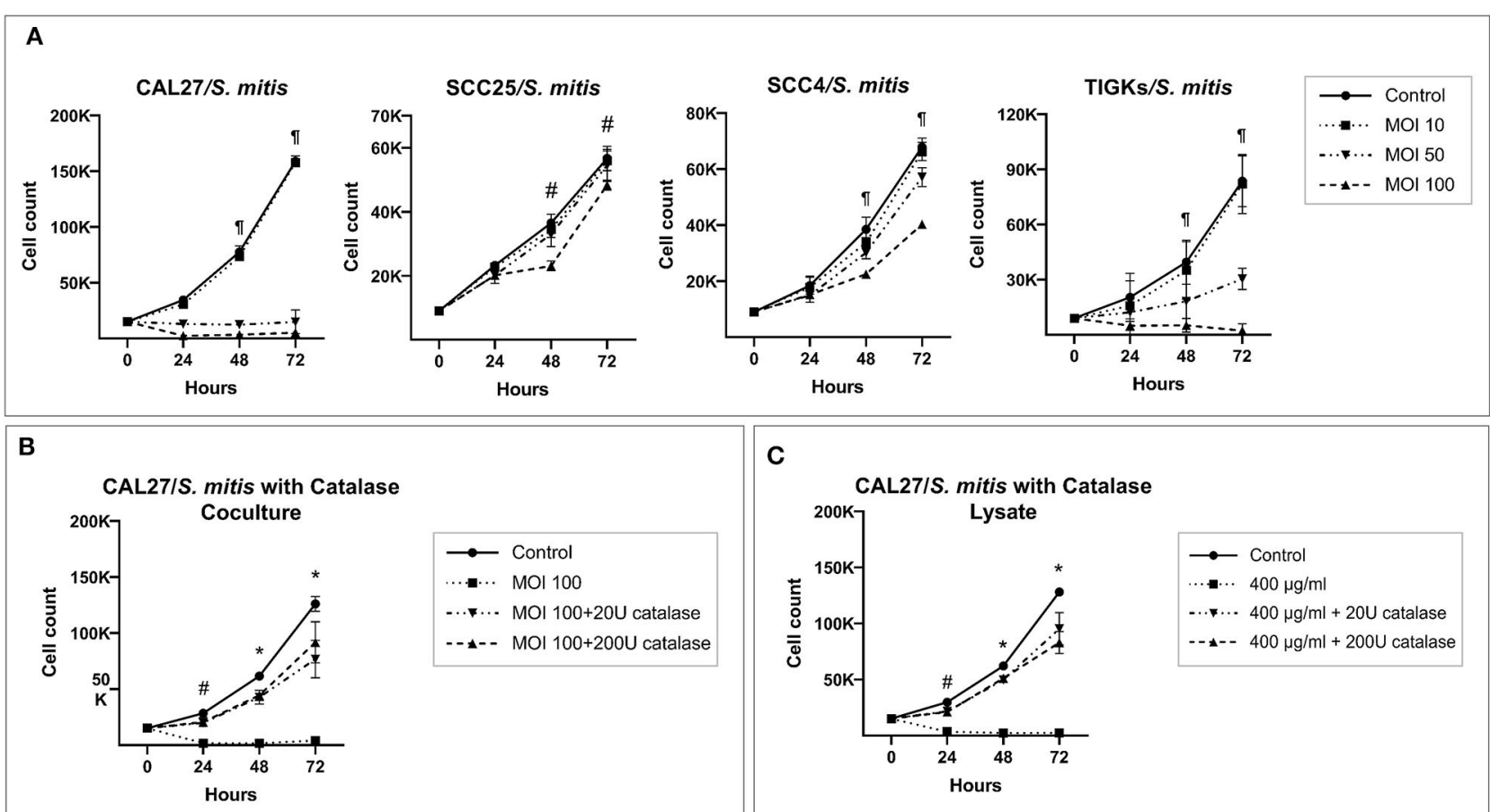

FIGURE 2 | Catalase inhibits cytotoxicity of S. mitis against oral cancer cell lines. (A) CAL27, SCC25, SCC4, and TIGKs (as non-cancer control) were cocultured with S. mitis at MOls of 10,50, and 100 and counting performed at 24,48 , and $72 \mathrm{~h}$. "Statistically significant $(p \leq 0.05)$ for MOl 50 and 100 compared to the control; \# for MOI 100 only. (B) CAL27 cocultured with S. mitis at MOI 100 in the presence or absence of exogenous catalase at 20 or $200 \mathrm{U}$. *Statistically significant $(p \leq 0.05)$ for all treatments compared to the control; \# for MOI 100 treatment without catalase. (C) Same as for (B) but using the lysate at $400 \mu \mathrm{g} / \mathrm{ml}$ instead. *Statistically significant for all treatments compared to the control; \# for $400 \mu \mathrm{g} / \mathrm{ml}$ lysate treatment without catalase.

explained in more detail below, were observed for CD36, IL6, and TNF- $\alpha$.

\section{CD36 Gene Expression Down-Regulated by Health Associated Species}

The effect of different bacteria on the expression of CD36 is shown in Figure 5. Lysates from the Gram-negative, health-associated bacteria, particularly $V$. parvula, caused a downregulation of CD36 in SCC25 and SCC4, whereas the lysate of $P$. gingivalis led to an upregulation in all cell lines, prominently in CAL27. The effects were mostly dosedependent. In cocultures, all health-associated species resulted in downregulation of CD36 in all cell lines (with the exception of $R$. mucilaginosa in CAL27); in most cases, the effect peaked at MOI 50. The effect of $P$. gingivalis varied with concentration, with the lowest MOI resulting in downregulation while the highest concentration associated with upregulation of CD36.

\section{Universal Upregulation of IL6 and TNF- $\alpha$}

With a few exceptions, all species, particularly the Gramnegatives, increased the expression of IL6 and TNF- $\alpha$ in a dose-dependent manner (Figures 6, 7). The upregulation was profound in SCC4. The effect of $H$. parainfluenzae, $N$. flavescens and $V$. parvula was consistent between the lysate and co-culture experiments. For $P$. gingivalis a notable upregulation was only observed in coculture, while the lysates did not result in changes in expression of the two genes in SCC4 and SCC25 and even downregulated them in CAL27. R. mucilaginosa upregulated TNF- $\alpha$, but hardly affected the expression of IL6. For S. mitis, the results varied by cell line and from the lysates to co-culture. To validate the results, we performed protein analysis of IL6 and TNF- $\alpha$ in supernatants from all the cell lines co-cultured with $H$. parainfluenzae, $N$. flavescens, $S$. mitis, and $P$. gingivalis at MOI 100 (Supplementary Method 7). We found the changes in protein levels to correlate with those identified by gene expression except for P. gingivalis (Supplementary Figure 24), for which IL6 and TNF- $\alpha$ (and all other proteins) tested low to nondetectable, probably due to break down by proteolytic enzymes produced by $P$. gingivalis.

\section{DISCUSSION}

The emerging role of microbiome in oral cancer is gaining much attention. The carcinogenic properties of oral pathogens like $P$. gingivalis and F. nucleatum are well-documented; however, not much is known about the role of health-associated commensals in oral cancer. In this study, we have attempted to understand the in-vitro effects of health-associated commensals on cell proliferation and expression of select genes in different OSCC cell lines. We tested both lysates and living bacteria to assess whether any effects are a result of bacterial components or products, or a 

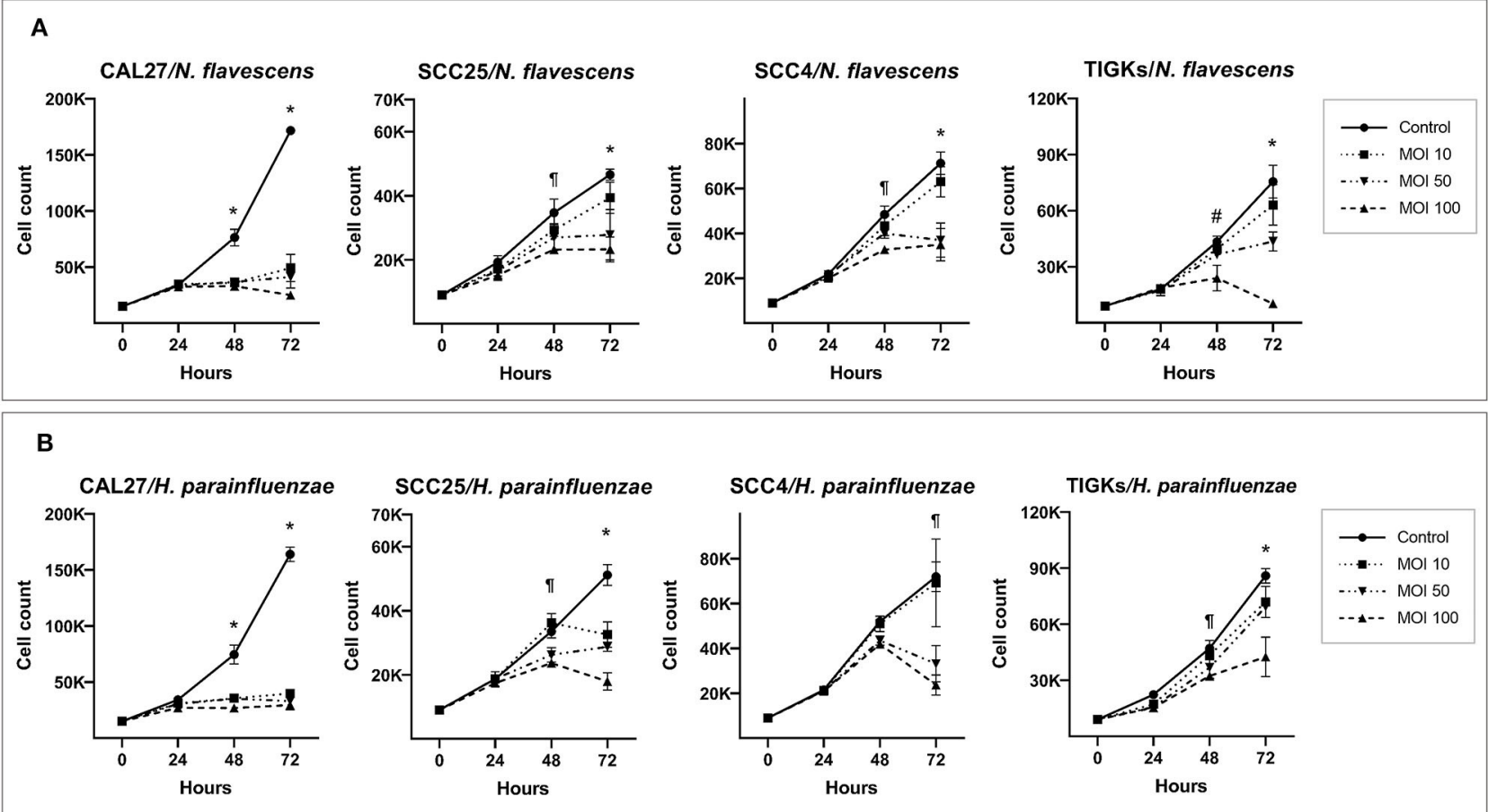

FIGURE $3 \mid$ H. parainfluenzae and N. flavescens cytotoxicity against oral cancer cell lines. CAL27, SCC25, SCC4, and TIGKs (as non-cancer control) were cocultured with (A) N. flavescens and (B) H. parainfluenzae at MOls of 10,50, and 100 and counting performed at 24,48 , and $72 \mathrm{~h}$. *Statistically significant $(p \leq 0.05)$ for all MOls against the control; Iffor MOI 50 and 100; \# for MOI 100 only. The corresponding results from the ATP assay are shown in Supplementary Figures 10-12.

\section{H. parainfluenzae (coculture)}

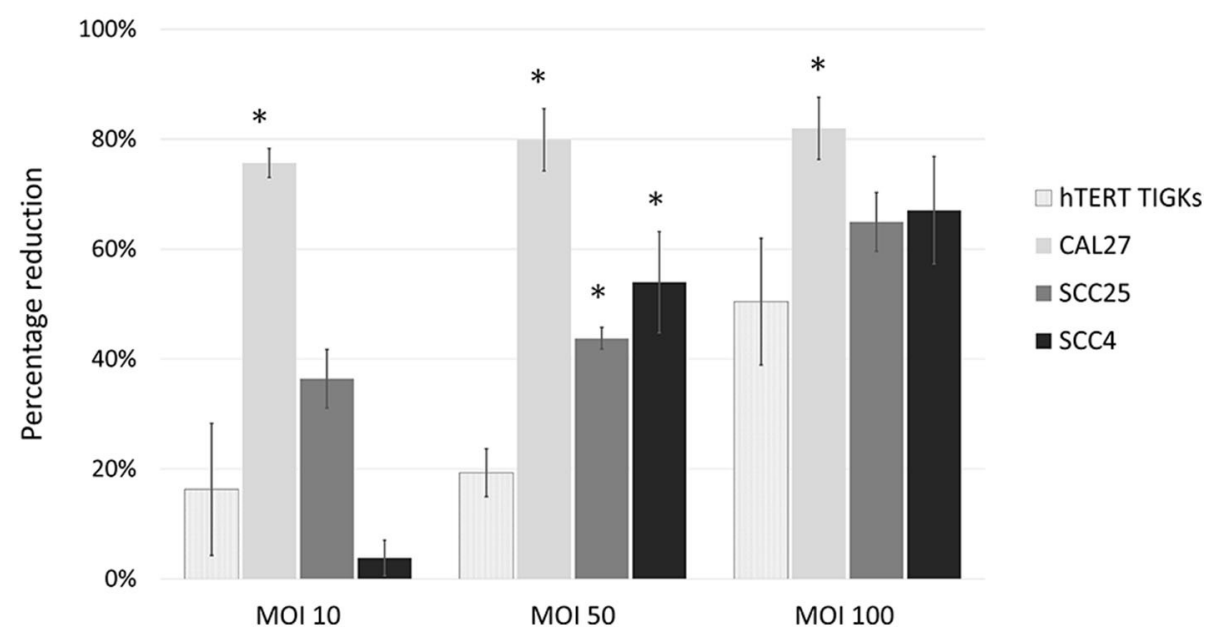

FIGURE 4 | Percentage reduction of cell proliferation by $H$. parainfluenzae in CAL27, SCC25, SCC4 and TIGKs in cocultures. *Statistically significant ( $0 \leq 0.05$ ) compared to TIGKs; pair-wise Student's t-test.

direct interaction between the bacteria and the tumor cells. For assessment of proliferation, we supplemented cell counting with ATP assay to improve reliability. In the co-culture experiments, we employed a novel approach in which sub-MIC concentration of antibiotics was used to allow bacterial cells to remain viable but without overgrowing. The purpose was to have the bacteria interact with cells for the entire duration of experiment (72 and $24 \mathrm{~h}$ for the proliferation and gene expression assays, respectively). Previous studies typically used short exposure time ( $45 \mathrm{~min}$ to $2 \mathrm{~h}$ ) to circumvent bacterial overgrowth in similar experiments (Hasegawa et al., 2007; Boonanantanasarn et al., 2012; Okahashi et al., 2014). Nevertheless, it is possible that 

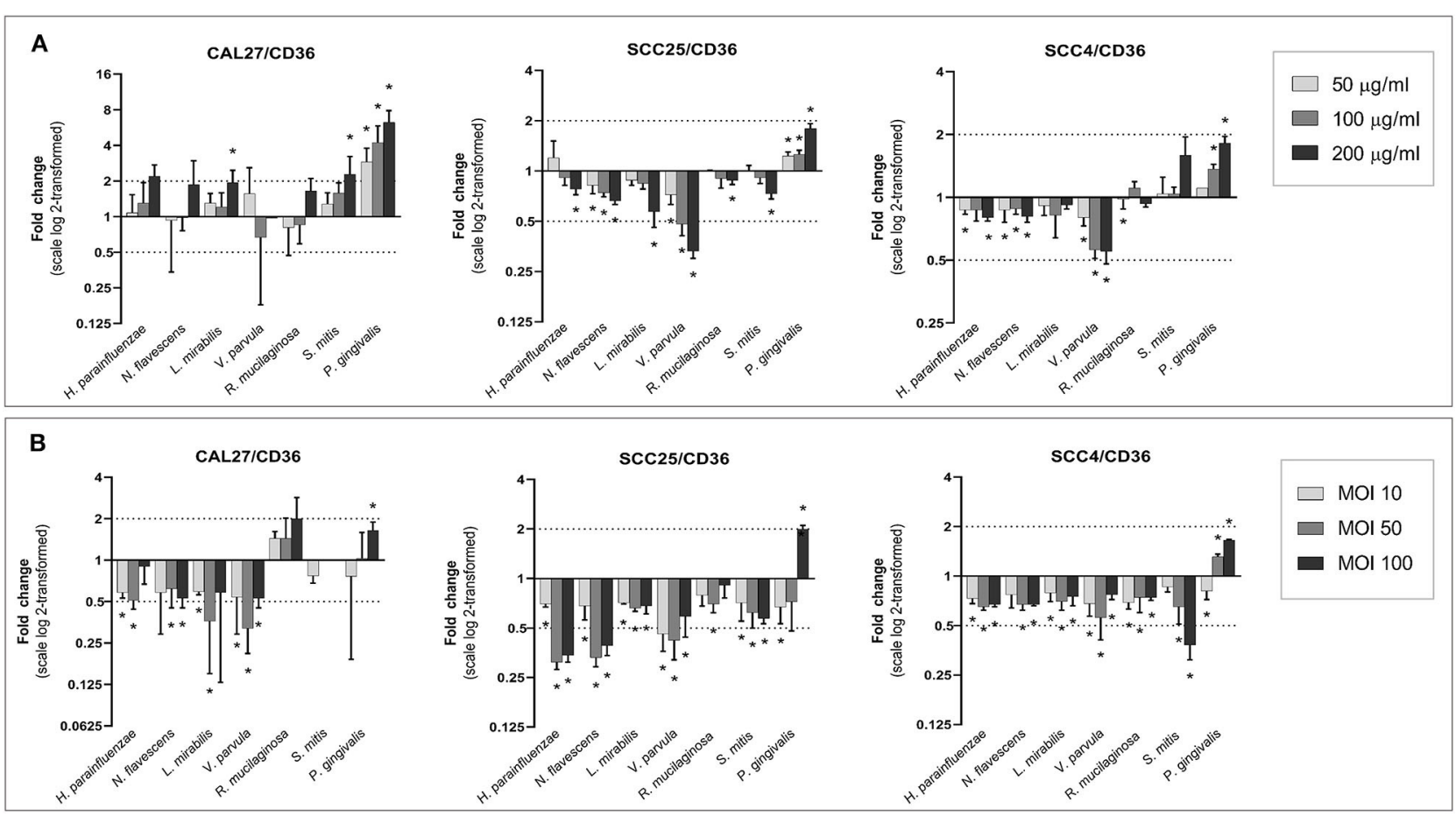

FIGURE 5 | Effect of the test bacteria on expression of CD36. CAL27, SCC25, and SCC4 were either (A) exposed to 50, 100, and 200 $\mu \mathrm{g} / \mathrm{ml}$ of each of the bacterial lysates, or (B) cocultured with each bacterium at MOls of 10, 50, and 100. RNA was extracted after $24 \mathrm{~h}$ and CD36 was quantified using one-step q-RT-PCR, using CASC3 as reference gene. *Statistically significant $(p \leq 0.05)$ compared to the non-treated control. Dashed line above and below horizontal axis indicate a 2-fold change.

antibiotics, even at sub-MIC levels, could have affected the bacterial cell wall structure and protein synthesis, which in turn, may explain, at least in part the differences in the results obtained with living cells and cell lysates.

Three species resulted in significant reduction of proliferation: S. mitis, N. flavescens and H. parainfluenzae. For S. mitis, the effects were observed with both the lysate as well as in co-culture. Several studies have shown it to be depleted in OSCC patients (Pushalkar et al., 2012; Schmidt et al., 2014; Al-Hebshi et al., 2017; Amer et al., 2017), and to negatively correlate with tumor staging (Yang et al., 2018), suggesting it may be playing a protective role against oral cancer. The cytotoxic effect observed in this study was inhibited by addition of catalase, indicating it was mediated by $\mathrm{H}_{2} \mathrm{O}_{2}$. This is not an entirely novel finding, given that the closely related species Streptococcus oralis and Streptococcus sanguinis have previously been shown to induce $\mathrm{H}_{2} \mathrm{O}_{2}$-mediated cytotoxicity in macrophages and epithelial cells (Okahashi et al., 2011, 2013, 2014). The addition of catalase, however, did not completely reverse the cytotoxic effects, suggesting that part of the cytotoxicity seen is $\mathrm{H}_{2} \mathrm{O}_{2}$-independent. Okahashi et al. (2014) observed the same for S. oralis. In contrast, and in total contradiction with these findings, Boonanantanasarn et al. (2012) demonstrated $\mathrm{H}_{2} \mathrm{O}_{2}$ produced by E. faecalis to enhance proliferation of oral cancer cell lines. One of the major reasons that can account for this drastic difference, is that the exposure of cells to bacteria and $\mathrm{H}_{2} \mathrm{O}_{2}$ in the latter study was limited to $45 \mathrm{~min}$, which is brief compared to our study.

$\mathrm{H}_{2} \mathrm{O}_{2}$ induces oxidative stress in mammalian cells which signals the activation of several cellular pathways including of pro-inflammatory cytokine production and cell death or apoptosis (Pelaia et al., 2004; Valko et al., 2007; Forman et al., 2010; Vilema-Enriquez et al., 2016; Park, 2018; Aggarwal et al., 2019). $\mathrm{H}_{2} \mathrm{O}_{2}$ acts as double-edged sword; while it is shown to be a potent carcinogen in normal cells (Lisanti et al., 2011), localized production of $\mathrm{H}_{2} \mathrm{O}_{2}$ has been used in hyperoxygenation therapy for cancer treatment (Liu and Wang, 2015; Vilema-Enriquez et al., 2016; Mast and Kuppusamy, 2018; Perillo et al., 2020). Studies have also shown that cancer cells are more sensitive to $\mathrm{H}_{2} \mathrm{O}_{2}$ than normal cells (Aykin-Burns et al., 2009) which has led to increased interest in exploring its therapeutic potential for cancer treatment including oral cancer. S. mitis is a core oral bacterial species, so the relevance of $\mathrm{H}_{2} \mathrm{O}_{2}$ it produces, along with related species, to oral cancer, or protection thereof, is worth further investigation.

Neisseria and Haemophilus spp. are ubiquitous oral commensals that have been found in lower abundance in OSCC patients compared to healthy controls (Al-Hebshi et al., 2017; Amer et al., 2017; Guerrero-Preston et al., 2017; Perera et al., 2017; Hayes et al., 2018). The two tested species in this study, $N$. flavescens and $H$. parainfluenzae, were found to induce cytotoxicity by intracellular infection. While the genera Neisseria 

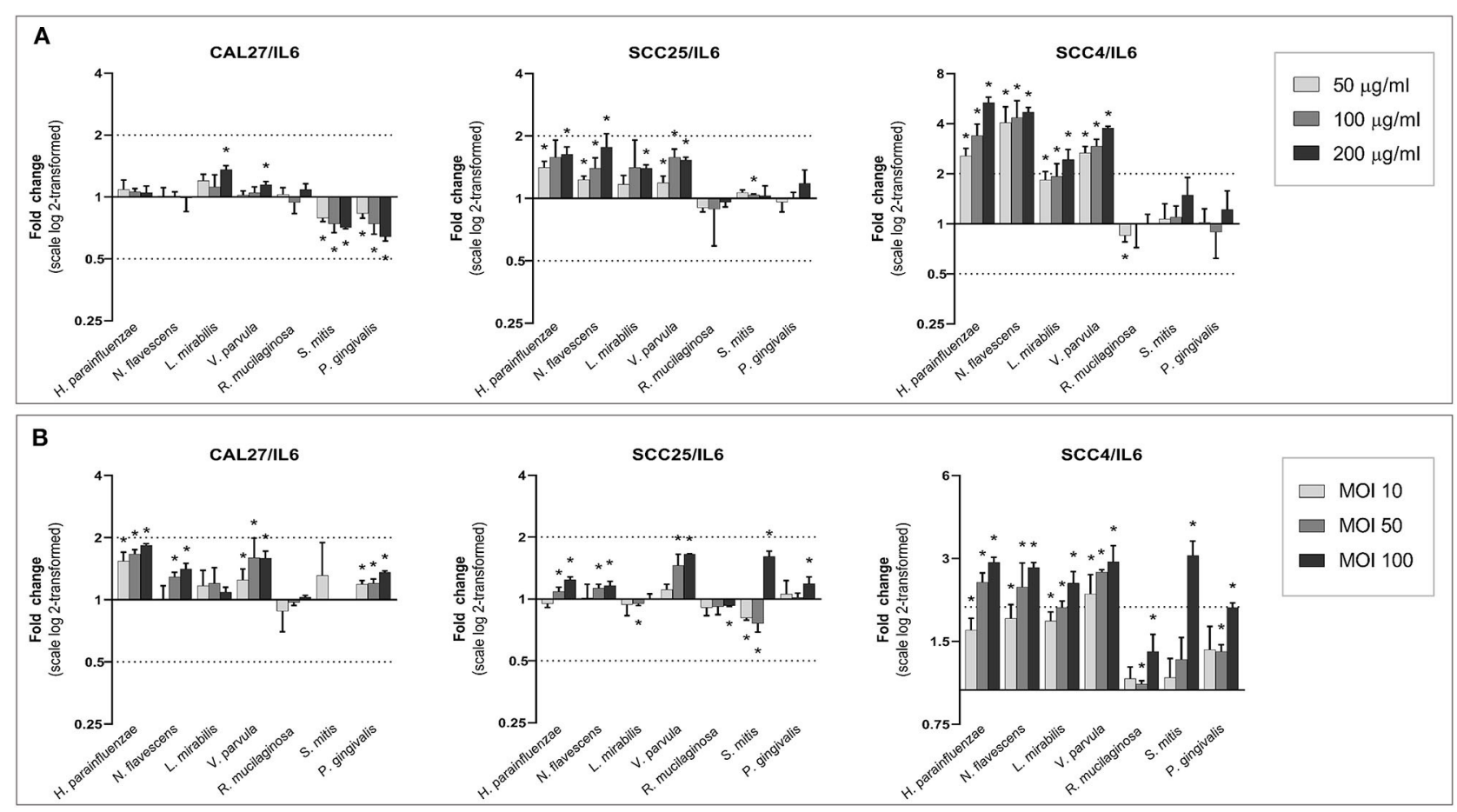

FIGURE 6 | Effect of the test bacteria on expression of IL6. CAL27, SCC25, and SCC4 were either (A) exposed to 50, 100, and $200 \mu \mathrm{g} / \mathrm{ml}$ of each of the bacterial lysates, or (B) cocultured with each bacterium at MOls of 10,50, and 100. RNA was extracted after $24 \mathrm{~h}$ and IL6 was quantified using one-step q-RT-PCR, using CASC3 as reference gene. *Statistically significantly compared to the non-treated control. Dashed line above and below horizontal axis indicate a 2-fold change.

and Haemophilus are known to be capable of causing intracellular infection as exemplified by Haemophilus influenzae and Neisseria meningiditis (Nikulin et al., 2006; Parisi and Martinez, 2014), this is the first report of internalization of $N$. flavescens and $H$. parainfluenzae per se. Interestingly, $H$. parainfluenzae showed significantly less cytotoxicity against non-cancer TIGK cells as compared to OSCC cells. Whether this may play a protective role against oral cancer, or can be exploited for prevention and/or treatment of oral cancer needs further investigation, for example by extending this work to in-vivo mouse models of oral cancer.

In the gene expression experiments, the results from lysate and coculture were largely consistent, suggesting most of the observed effects do not involve direct interaction between the bacteria and host cell. The only exception was $P$. gingivalis for which the results substantially differed between the lysate and coculture particularly of IL6 and TNF- $\alpha$, that were notably upregulated only in coculture, suggesting internalization of $P$. gingivalis is important for these effects. Three genes were prominently modulated by the different species: CD36, IL6, and TNF- $\alpha$. CD36 is an important transmembrane protein receptor which involved in regulating immune response, cell adhesion and lipid metabolism. CD36 plays an important role in cancer by accelerating tumor growth and metastasis, and modulating the immune response and response to therapy, and has thus been a target for cancer treatment (Wang and Li, 2019). Recently, Sakurai et al. (2020) have demonstrated the role of CD36 in facilitating the proliferation and migration activity of
OSCC cells, possibly by inhibiting $\beta$-catenin signaling pathway, and they emphasized its usefulness in the diagnosis of highgrade tumor and targeted therapy of oral cancer. Interestingly, the health-associated species in this study, particularly the Gram-negatives, showed significant downregulation in CD36 expression in contrast with the pathogenic control $P$. gingivalis that upregulated it. This is an important finding given the key role of CD36 in cancer. The possibility of targeting it with these oral health-associated species should be explored in future studies.

TNF- $\alpha$ and IL6 are important pro-inflammatory cytokines known to have pleotropic function in immune response, inflammation, cell death and tumor progression. In our study, significant upregulation of TNF- $\alpha$ and IL6 was induced by both health-associated species as well as $P$. gingivalis. However, that does not necessarily mean the upregulation is associated with same biological outcomes. Both TNF- $\alpha$ and IL6 are known to have complex effects and dual roles in cancer (Bertazza and Mocellin, 2010; Fisher et al., 2014). Some studies have shown TNF- $\alpha$ to possess anti-tumor properties and can be exploited for treatment of some cancer types, while others have demonstrated it contributes to tumor progression by modulation of the immune response (Montfort et al., 2019). The overall effect seems to be dependent on its level, with high concentrations favoring tumor regression. Similarly, increased expression of IL6 has been associated with tumor progression and metastasis but, at the same time, it is shown to augment adaptive immunity against tumor growth (Fisher et al., 2014). 

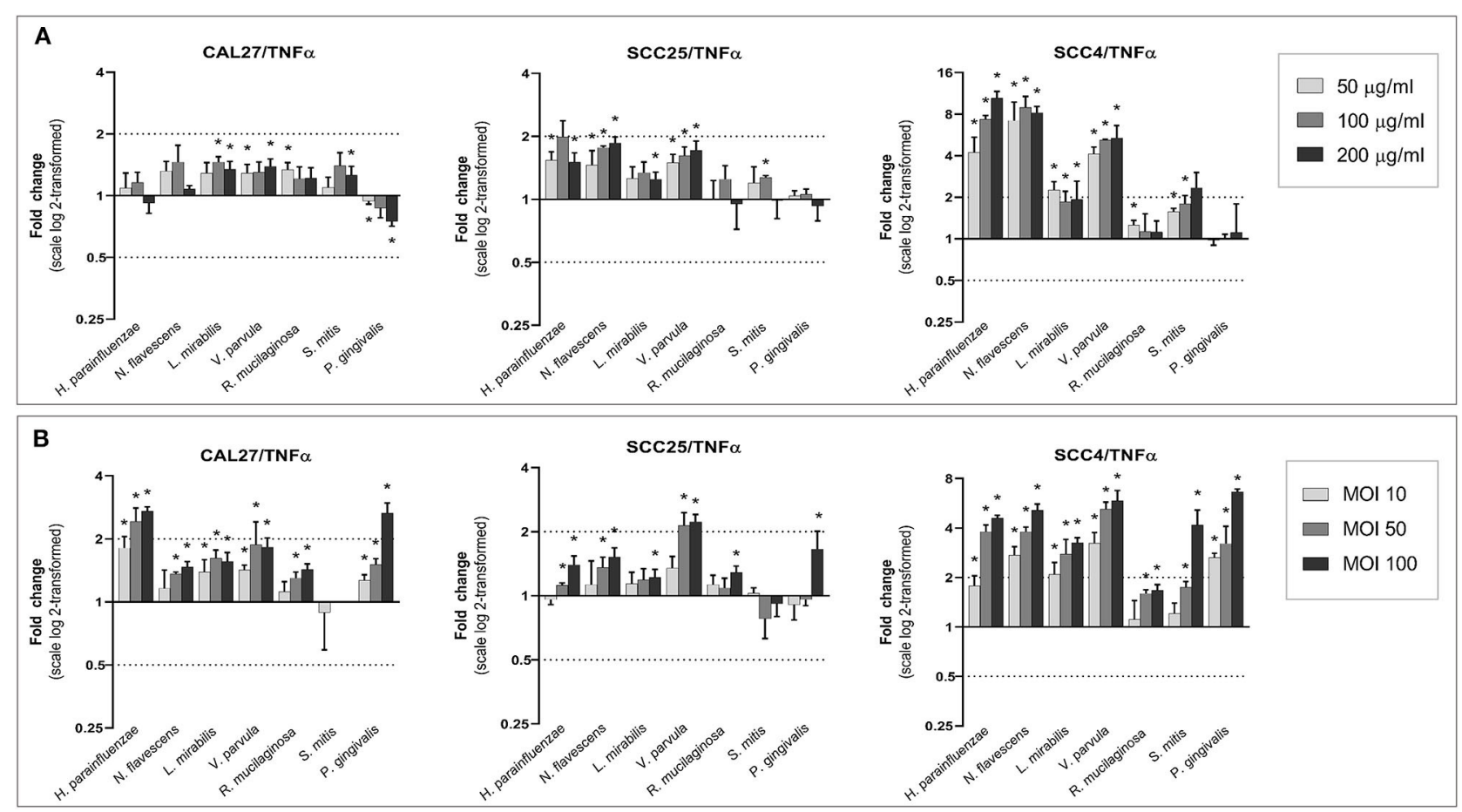

FIGURE 7 | Effect of the test bacteria on expression of TNF $\alpha$. CAL27, SCC25, and SCC4 were either (A) exposed to 50, 100, and $200 \mu \mathrm{g} / \mathrm{ml}$ of each of the bacterial lysates, or (B) cocultured with each bacterium at MOls of 10, 50, and 100. RNA was extracted after $24 \mathrm{~h}$ and TNF $\alpha$ was quantified using one-step q-RT-PCR, using CASC3 as reference gene. *Statistically significant compared to the non-treated control. Dashed line above and below horizontal axis indicate a 2 -fold change.

It has been emphasized that the biological outcome of these cytokines depends on multiple factors including cell type, concentration, tissue condition, tumor microenvironment, and cross talk between several signaling pathways activated at a particular time (Fisher et al., 2014; Montfort et al., 2019). We believe that further studies focusing on global transcriptome profiling are needed to get better understanding of biological mechanisms activated by commensals in OSCC cell lines.

One of the interesting findings of the study is that different OSCC lines exhibited distinct responses. This was probably due to differences in the expression of surface receptors between the three cell lines. For example, it has been shown that SCC4 has lower expression of TLR4 compared to CAL27 (He et al., 2015). The cells also have different doubling time, with CAL27 being the fastest, which probably explains it was more susceptible to the cytotoxic effects of the different bacteria.

While the bacterial species that resulted in pronounced reduction in proliferation were also tested on TIGKs as noncancer cell control, it should be noted that immortalized cells bypass senescence similar to cancer cells, and most cancer cells also show an increased expression of hTERT proteins (Boehm and Hahn, 2004; Zhang et al., 2016). Therefore, it may have been more reliable to use primary gingival keratinocytes for assessing differential effects of the bacterial species on cancer vs. noncancer cells. Another limitation of our study is that the expression of marker genes was restricted to only a single time point $(24 \mathrm{~h})$ due to logistical constraints, namely the large-scale nature of the experiments. It would have been interesting to follow the change in gene expression at also 48 and $72 \mathrm{~h}$. Moreover, in addition to proliferation and gene expression of marker gene, it may have been useful to perform some assays of DNA damage and apoptosis to get more mechanistic insights, but again, that was not feasible given it was primarily a screening study.

Furthermore, considering the exploratory nature of this study, we used only six marker genes to screen the possible changes at molecular level, but there are probably many more genes that have been affected. This, however, can be addressed using a high throughput approach like RNA Seq or microarray analysis in a future study. In this in-vitro study, we assessed the effect of single species on each cell line, but in in vivo conditions, the tumor is exposed to a microbial consortium, so for future studies it may be important to use mixtures of oral bacterial species. Finally, in vivo, bacteria do not only interact with the tumor cells but also with their microenvironment that is known to play an important role in modulation of oral cancer, which highlights the importance of taking this work further to animal models of oral cancer.

In conclusion, this is the first study to perform large-scale, in vitro screening for the effect of oral health-associated bacteria on OSCC cell lines. This was required to fill in some of the knowledge gap in this area, and to form the basis and generate hypotheses for future work. Indeed, a number of interesting findings were revealed. Particularly the differential cytotoxicity by $H$. parainfluenzae against the oral cancer cell lines and the 
downregulation of CD36 by the health-associated species, and the possibility of exploiting them for prevention or/and treatment of oral cancer, are worth further investigation. Also, profiling of the global transcriptome rather than select genes is needed to provide a better and comprehensive understanding of how oral health-associated species may modulate the behavior of oral cancer cells.

\section{DATA AVAILABILITY STATEMENT}

The raw data supporting the conclusions of this article will be made available by the authors, without undue reservation.

\section{AUTHOR CONTRIBUTIONS}

NNA conceived and designed the study. DB, MY, VT, and SP assisted in the design of experiments. DB conducted and performed the majority of the experiments and data entry. VT, $\mathrm{RL}$, and LV assisted with lab work and provided technical advice.

\section{REFERENCES}

Aggarwal, V., Tuli, H. S., Varol, A., Thakral, F., Yerer, M. B., Sak, K., et al. (2019). Role of reactive oxygen species in cancer progression: molecular mechanisms and recent advancements. Biomolecules 9:735. doi: 10.3390/biom 9110735

Al-Hebshi, N. N., Borgnakke, W. S., and Johnson, N. W. (2019). The microbiome of oral squamous cell carcinomas: a functional perspective. Curr. Oral Health Rep. 6, 145-160. doi: 10.1007/s40496-019-0215-5

Al-Hebshi, N. N., Nasher, A. T., Maryoud, M. Y., Homeida, H. E., Chen, T., Idris, A. M., et al. (2017). Inflammatory bacteriome featuring Fusobacterium nucleatum and Pseudomonas aeruginosa identified in association with oral squamous cell carcinoma. Sci. Rep. 7:1834. doi: 10.1038/s41598-017-02079-3

Amer, A., Galvin, S., Healy, C. M., and Moran, G. P. (2017). The microbiome of potentially malignant oral leukoplakia exhibits enrichment for fusobacterium, leptotrichia, campylobacter, and rothia species. Front. Microbiol. 8:2391. doi: $10.3389 /$ fmicb. 2017.02391

Aykin-Burns, N., Ahmad, I. M., Zhu, Y., Oberley, L. W., and Spitz, D. R. (2009). Increased levels of superoxide and $\mathrm{H} 2 \mathrm{O} 2$ mediate the differential susceptibility of cancer cells versus normal cells to glucose deprivation. Biochem. J. 418, 29-37. doi: 10.1042/BJ20081258

Benjamini, Y., Krieger, A. M., and Yekutieli, D. (2006). Adaptive linear stepup procedures that control the false discovery rate. Biometrika 93, 491-507. doi: 10.1093/biomet/93.3.491

Bertazza, L., and Mocellin, S. (2010). The dual role of tumor necrosis factor (TNF) in cancer biology. Curr. Med. Chem. 17, 3337-3352. doi: 10.2174/092986710793176339

Boehm, J. S., and Hahn, W. C. (2004). Immortalized cells as experimental models to study cancer. Cytotechnology 45, 47-59. doi: 10.1007/s10616-004-5125-1

Boonanantanasarn, K., Gill, A. L., Yap, Y., Jayaprakash, V., Sullivan, M. A., and Gill, S. R. (2012). Enterococcus faecalis enhances cell proliferation through hydrogen peroxide-mediated epidermal growth factor receptor activation. Infect. Immun. 80, 3545-3558. doi: 10.1128/IAI.00479-12

Bray, F., Ferlay, J., Soerjomataram, I., Siegel, R. L., Torre, L. A., and Jemal, A. (2018). Global cancer statistics 2018: GLOBOCAN estimates of incidence and mortality worldwide for 36 cancers in 185 countries. CA Cancer J. Clin. 68, 394-424. doi: 10.3322/caac. 21492

Chocolatewala, N., Chaturvedi, P., and Desale, R. (2010). The role of bacteria in oral cancer. Indian J. Med. Paediatr. Oncol. 31, 126-131. doi: 10.4103/0971-5851.76195
NNA and DB performed data analysis. NNA, DB, and VJ drafted the manuscript. All authors read and approved the final version of the article.

\section{FUNDING}

This study was funded by the Pennsylvania Commonwealth Universal Research Enhancement Program (CURE, grant\# 4100079747).

\section{ACKNOWLEDGMENTS}

We would like to thank Profs. Thomas Rams and Bettina Buttaro for their useful suggestions.

\section{SUPPLEMENTARY MATERIAL}

The Supplementary Material for this article can be found online at: https://www.frontiersin.org/articles/10.3389/fcimb. 2020.575656/full\#supplementary-material

Dunnett, C. W. (1955). A multiple comparison procedure for comparing several treatments with a control. J. Am. Stat. Assoc. 50, 1096-1121. doi: 10.1080/01621459.1955.10501294

Fisher, D. T., Appenheimer, M. M., and Evans, S. S. (2014). The two faces of IL-6 in the tumor microenvironment. Semin. Immunol. 26, 38-47. doi: 10.1016/j.smim.2014.01.008

Fitzsimonds, Z. R., Rodriguez-Hernandez, C. J., Bagaitkar, J., and Lamont, R. J. (2020). From beyond the pale to the pale riders: the emerging association of bacteria with oral cancer. J. Dent. Res. 99, 604-612. doi: $10.1177 / 0022034520907341$

Forman, H. J., Maiorino, M., and Ursini, F. (2010). Signaling functions of reactive oxygen species. Biochemistry 49, 835-842. doi: 10.1021/bi9020378

Guerrero-Preston, R., White, J. R., Godoy-Vitorino, F., Rodriguez-Hilario, A., Navarro, K., Gonzalez, H., et al. (2017). High-resolution microbiome profiling uncovers Fusobacterium nucleatum, Lactobacillus gasseri/johnsonii, and Lactobacillus vaginalis associated to oral and oropharyngeal cancer in saliva from HPV positive and HPV negative patients treated with surgery and chemoradiation. Oncotarget 8, 110931-110948. doi: 10.18632/oncotarget.20677

Gupta, B., Johnson, N. W., and Kumar, N. (2016). Global epidemiology of head and neck cancers: a continuing challenge. Oncology 91, 13-23. doi: $10.1159 / 000446117$

Hasegawa, Y., Mans, J. J., Mao, S., Lopez, M. C., Baker, H. V., Handfield, M., et al. (2007). Gingival epithelial cell transcriptional responses to commensal and opportunistic oral microbial species. Infect. Immun. 75, 2540-2547. doi: 10.1128/IAI.01957-06

Hayes, R. B., Ahn, J., Fan, X., Peters, B. A., Ma, Y., Yang, L., et al. (2018). Association of oral microbiome with risk for incident head and neck squamous cell cancer. JAMA Oncol. 4, 358-365. doi: 10.1001/jamaoncol.2017.4777

He, Z., Deng, R., Huang, X., Ni, Y., Yang, X., Wang, Z., et al. (2015). Lipopolysaccharide enhances OSCC migration by promoting epithelial-mesenchymal transition. J. Oral Pathol. Med. 44, 685-692. doi: 10.1111/jop. 12285

Lingen, M. W., Xiao, W., Schmitt, A., Jiang, B., Pickard, R., Kreinbrink, P., et al. (2013). Low etiologic fraction for high-risk human papillomavirus in oral cavity squamous cell carcinomas. Oral Oncol. 49, 1-8. doi: 10.1016/j.oraloncology.2012.07.002

Lisanti, M. P., Martinez-Outschoorn, U. E., Lin, Z., Pavlides, S., WhitakerMenezes, D., Pestell, R. G., et al. (2011). Hydrogen peroxide fuels aging, inflammation, cancer metabolism and metastasis: the seed and soil also needs "fertilizer". Cell Cycle 10, 2440-2449. doi: 10.4161/cc.10.15.16870 
Liu, J., and Wang, Z. (2015). Increased oxidative stress as a selective anticancer therapy. Oxid. Med. Cell. Longev. 2015:294303. doi: 10.1155/2015/294303

Livak, K. J., and Schmittgen, T. D. (2001). Analysis of relative gene expression data using real-time quantitative PCR and the 2(-Delta Delta C(T)) Method. Methods 25, 402-408. doi: 10.1006/meth.2001.1262

Mast, J. M., and Kuppusamy, P. (2018). Hyperoxygenation as a therapeutic supplement for treatment of triple negative breast cancer. Front. Oncol. 8:527. doi: 10.3389/fonc.2018.00527

Montfort, A., Colacios, C., Levade, T., Andrieu-Abadie, N., Meyer, N., and Segui, B. (2019). The TNF paradox in cancer progression and immunotherapy. Front. Immunol. 10:1818. doi: 10.3389/fimmu.2019.01818

Mukherjee, P. K., Wang, H., Retuerto, M., Zhang, H., Burkey, B., Ghannoum, M. A., et al. (2017). Bacteriome and mycobiome associations in oral tongue cancer. Oncotarget 8, 97273-97289. doi: 10.18632/oncotarget.21921

Nikulin, J., Panzner, U., Frosch, M., and Schubert-Unkmeir, A. (2006). Intracellular survival and replication of Neisseria meningitidis in human brain microvascular endothelial cells. Int. J. Med. Microbiol. 296, 553-558. doi: 10.1016/j.ijmm.2006.06.006

Ohshima, J., Wang, Q., Fitzsimonds, Z. R., Miller, D. P., Sztukowska, M. N., Jung, Y. J., et al. (2019). Streptococcus gordonii programs epithelial cells to resist ZEB2 induction by Porphyromonas gingivalis. Proc. Natl. Acad. Sci. U.S.A. 116, 8544-8553. doi: 10.1073/pnas.1900101116

Okahashi, N., Nakata, M., Sumitomo, T., Terao, Y., and Kawabata, S. (2013). Hydrogen peroxide produced by oral Streptococci induces macrophage cell death. PLoS ONE 8:e62563. doi: 10.1371/journal.pone.0062563

Okahashi, N., Okinaga, T., Sakurai, A., Terao, Y., Nakata, M., Nakashima, K., et al. (2011). Streptococcus sanguinis induces foam cell formation and cell death of macrophages in association with production of reactive oxygen species. FEMS Microbiol. Lett. 323, 164-170. doi: 10.1111/j.1574-6968.2011.02375.x

Okahashi, N., Sumitomo, T., Nakata, M., Sakurai, A., Kuwata, H., and Kawabata, S. (2014). Hydrogen peroxide contributes to the epithelial cell death induced by the oral mitis group of streptococci. PLOS ONE 9:e88136. doi: 10.1371/journal.pone.0088136

Parisi, D. N., and Martinez, L. R. (2014). Intracellular Haemophilus influenzae invades the brain: is zyxin a critical blood brain barrier component regulated by TNF- $\alpha$ ? Virulence 5, 645-647. doi: 10.4161/viru.36086

Park, W. H. (2018). Hydrogen peroxide inhibits the growth of lung cancer cells via the induction of cell death and G1phase arrest. Oncol. Rep. 40, 1787-1794. doi: 10.3892/or.2018.6535

Pelaia, G., Cuda, G., Vatrella, A., Gallelli, L., Fratto, D., Gioffre, V., et al. (2004). Effects of hydrogen peroxide on MAPK activation, IL-8 production and cell viability in primary cultures of human bronchial epithelial cells. J. Cell. Biochem. 93, 142-152. doi: 10.1002/jcb.20124

Perera, M., Al-Hebshi, N. N., Perera, I., Ipe, D., Ulett, G. C., Speicher, D. J., et al. (2017). A dysbiotic mycobiome dominated by Candida albicans is identified within oral squamous-cell carcinomas. J. Oral Microbiol. 9:1385369. doi: 10.1080/20002297.2017.1385369

Perera, M., Al-Hebshi, N. N., Speicher, D. J., Perera, I., and Johnson, N. W. (2016). Emerging role of bacteria in oral carcinogenesis: a review with special reference to perio-pathogenic bacteria. J. Oral Microbiol. 8:32762. doi: 10.3402/jom.v8.32762

Perillo, B., Di Donato, M., Pezone, A., Di Zazzo, E., Giovannelli, P., Galasso, G., et al. (2020). ROS in cancer therapy: the bright side of the moon. Exp. Mol. Med. 52, 192-203. doi: 10.1038/s12276-020-0384-2

Pushalkar, S., Ji, X., Li, Y., Estilo, C., Yegnanarayana, R., Singh, B., et al. (2012). Comparison of oral microbiota in tumor and non-tumor tissues of patients with oral squamous cell carcinoma. BMC Microbiol. 12:144. doi: 10.1186/1471-2180-12-144

Sakurai, K., Tomihara, K., Yamazaki, M., Heshiki, W., Moniruzzaman, R., Sekido, K., et al. (2020). CD36 expression on oral squamous cell carcinoma cells correlates with enhanced proliferation and migratory activity. Oral Dis. 26, 745-755. doi: 10.1111/odi.13210

Schmidt, B. L., Kuczynski, J., Bhattacharya, A., Huey, B., Corby, P. M., Queiroz, E. L., et al. (2014). Changes in abundance of oral microbiota associated with oral cancer. PLoS ONE 9:e98741. doi: 10.1371/journal.pone.0098741

Siegel, R. L., Miller, K. D., and Jemal, A. (2017). Cancer Statistics, 2017. CA Cancer J. Clin. 67, 7-30. doi: 10.3322/caac. 21387

Valko, M., Leibfritz, D., Moncol, J., Cronin, M. T., Mazur, M., and Telser, J. (2007). Free radicals and antioxidants in normal physiological functions and human disease. Int. J. Biochem. Cell Biol. 39, 44-84. doi: 10.1016/j.biocel.2006.07.001

Vilema-Enriquez, G., Arroyo, A., Grijalva, M., Amador-Zafra, R. I., and Camacho, J. (2016). Molecular and cellular effects of hydrogen peroxide on human lung cancer cells: potential therapeutic implications. Oxid. Med. Cell. Longev. 2016:1908164. doi: 10.1155/2016/1908164

Wang, J., and Li, Y. (2019). CD36 tango in cancer: signaling pathways and functions. Theranostics 9, 4893-4908. doi: 10.7150/thno.36037

Yang, C. Y., Yeh, Y. M., Yu, H. Y., Chin, C. Y., Hsu, C. W., Liu, H., et al. (2018). Oral microbiota community dynamics associated with oral squamous cell carcinoma staging. Front. Microbiol. 9:862. doi: 10.3389/fmicb.2018. 00862

Zhang, N., Xie, Y., Tai, Y., Gao, Y., Guo, W., Yu, W., et al. (2016). Bufalin inhibits hTERT expression and colorectal cancer cell growth by targeting CPSF4. Cell. Physiol. Biochem. 40, 1559-1569. doi: 10.1159/000453206

Zhao, H., Chu, M., Huang, Z., Yang, X., Ran, S., Hu, B., et al. (2017). Variations in oral microbiota associated with oral cancer. Sci. Rep. 7:11773. doi: 10.1038/s41598-017-11779-9

Conflict of Interest: The authors declare that the research was conducted in the absence of any commercial or financial relationships that could be construed as a potential conflict of interest.

Copyright (C) 2020 Baraniya, Jain, Lucarelli, Tam, Vanderveer, Puri, Yang and Alhebshi. This is an open-access article distributed under the terms of the Creative Commons Attribution License (CC BY). The use, distribution or reproduction in other forums is permitted, provided the original author(s) and the copyright owner(s) are credited and that the original publication in this journal is cited, in accordance with accepted academic practice. No use, distribution or reproduction is permitted which does not comply with these terms. 